\title{
Evaluación de la capacidad de reacción de coliformes de aguas residuales a la fotocatálisis con técnicas de recubrimiento por inmersión
}

DOI: https://doi.org/10.33262/ap.v3i3.1.102

\begin{abstract}
(c) (1) (2) (2)
Evaluation of the reaction capacity of wastewater coliforms to photocatalysis with dip coating techniques

Sofía Carolina Godoy Ponce, ${ }^{1}$ Juan Carlos González García. ${ }^{2}$, José Gerardo León Chimbolema. ${ }^{3} \&$ Erika Daniela Castillo Hidalgo. ${ }^{4}$
\end{abstract}

\begin{abstract}
Introduction: The presence of fecal and total coliforms in wastewater are indicators of water quality and the degree of exposure and impact of human activities on it. Objective: Apply photocatalysis processes with sunlight and the Dip - Coating method for the reduction of coliforms in wastewater. Objective: Apply photocatalysis processes with sunlight and the Dip - Coating method for the reduction of coliforms in wastewater. Methodology: The process included perpendicular immersion and subsequent extraction of the substrate at a controlled speed in a solution prepared for the formation of the Titanium Dioxide film, drainage in the same equipment, evaporation and drying known as the DIP_coating method together with the solar disinfection of the water (SODIS) that used solar energy to destroy pathogenic microorganisms that cause water-borne diseases,

1 Docente Investigador, Carrera de Ingeniería Ambiental, Facultad de Ciencias, Escuela Superior Politécnica de Chimborazo - ESPOCH. Riobamba, sofia.godoy@espoch.edu.ec ORCID: https://orcid.org/0000-0002-6479-4343

2 Docente Investigador, Carrera de Ingeniería Ambiental, Facultad de Ciencias, Escuela Superior Politécnica de Chimborazo - ESPOCH. Riobamba, juan.gonzalez@espoch.edu.ec ORCID: https://orcid.org/0000-0001-9066-1600

3 Docente Investigador, Carrera de Ingeniería Ambiental, Facultad de Ciencias, Escuela Superior Politécnica de Chimborazo - ESPOCH. Riobamba, gerardo.leon@espoch.edu.ec ORCID: https://orcid.org/0000-0001-9202-8542

${ }^{4}$ Investigador independiente, latieridani@ hotmail.com ORCID: https://orcid.org/0000-0002-2756-3810
\end{abstract}


relating UV-A radiation, which had a germicidal effect, and infrared radiation, responsible for raising the temperature of the water. The combined use of UV-A radiation and heat produced a synergistic effect that increased the efficiency of the process. Four experimental units were used: two were applied by the SODIS method and the other two applied SODIS with TIO2 in the Dip-coating method. The residual water samples were placed in PET plastic bottles prepared in three solutions $\left(10^{-1}, 10^{-2}\right.$ and $\left.10^{-3}\right)$, performing the microbiological analysis_quantification of coliforms in Compact Dry EC plates incubated for 24 hours at $35^{\circ} \mathrm{C}$. Results: A significant reduction was evidenced, especially in the $10^{-3}$ dilution, accounting for the case of fecal coliforms in the SODIS method: $3900 \mathrm{MPN} / 100 \mathrm{ml}$ and DIP-COATING: $100 \mathrm{MPN} / 100 \mathrm{ml}$; and for total coliforms in SODIS: 12500 NMP / 100ml and DIP-COATING: 1100 NMP / 100ml, at optimal treatment conditions of $180 \mathrm{~min}$ at $42{ }^{\circ} \mathrm{C}$. Conclusions: the exposed conditions and suitable climatic conditions give the maximum reduction effect by combining the two SODIS / DIP-Coating methodologies where the photocatalysis process with sunlight microbiologically improved the water quality where titanium dioxide was a low-cost semiconductor, easy accessibility, low toxicity, resistance to photo corrosion and catalytic efficiency

Keywords: Sodis, Dip-coating, fecal and total coliforms, waste water

\section{Resumen.}

Introducción: La presencia de coliformes fecales y totales en aguas residuales constituyen indicadores de la calidad del agua y del grado de exposición e impacto de las actividades humanas en ella. Objetivo: Aplicar procesos de fotocatálisis con luz solar y el método Dip - Coating para la reducción de coliformes de aguas residuales. Metodología: El proceso incluyó la inmersión perpendicular y posterior extracción del sustrato a velocidad controlada en una solución preparada para la formación de la película de Dióxido de Titanio, drenaje en el mismo equipo, evaporación y secado conocido como método DIP_coating conjuntamente con la desinfección solar del agua (SODIS) que usó la energía solar para destruir los microorganismos patógenos que causan enfermedades transmitidas por el agua, relacionando la radiación UV-A, que tuvo efecto germicida y la radiación infrarroja, encargada de elevar la temperatura del agua. El uso combinado de la radiación UV-A y del calor produjo un efecto de sinergia que incrementó la eficacia del proceso. Se trabajaron cuatro unidades experimentales: dos fueron aplicadas por el método SODIS y las otras dos aplicaron SODIS con $\mathrm{TIO}_{2}$ en el método Dip-coating. Las muestras de agua residual fueron dispuestas en botellas de plástico PET preparadas en tres disoluciones $\left(10^{-1}, 10^{-2}\right.$ y $\left.10^{-3}\right)$ realizándose el análisis microbiológico_cuantificación de coliformes en placas Compact Dry EC incubadas 24 horas a $35^{\circ} \mathrm{C}$. Resultados: Se evidenció una reducción significativa, sobre todo en la dilución $10^{-3}$, llegando a contabilizar para el caso de coliformes fecales en el método SODIS: 3900 NMP/100ml y DIP-COATING: $100 \mathrm{NMP} / 100 \mathrm{ml}$; y para coliformes totales en SODIS: 12500 NMP/100ml y DIP-COATING: 1100 NMP/100ml, a condiciones óptimas de tratamiento de 180 min a $42^{\circ} \mathrm{C}$. Conclusiones: las condiciones expuestas y condiciones climáticas adecuadas se da el efecto de reducción máxima combinando las dos metodologías 
SODIS/DIP-Coating donde el proceso de fotocatálisis con luz solar mejoró microbiológicamente la calidad del agua donde el dióxido de titanio fue un semiconductor de bajo costo, fácil accesibilidad, baja toxicidad, resistencia a la foto corrosión y eficiencia catalítica.

Palabras claves: Sodis, Dip-coating, coliformes fecales y totales, agua residual

\section{Introducción}

En la actualidad, el cuidado y la protección de los cuerpos de agua dulce se ha convertido en una tarea de primera línea en la agenda de países y organismos multilaterales como las Naciones Unidas (ONU), por ser una problemática que está directamente relacionada con el desarrollo y la salud humana (Restrepo et al., 2008). A partir del siglo XX la interacción de la materia con la luz se realiza desde el punto de vista microscópico, considerándola como un proceso de absorción de un fotón que conlleva la creación de un par electrónhueco o la emisión o destrucción de un fotón creando excitaciones. La materia puede interactuar con la luz en un amplio rango de longitudes de onda y con diversos cambios en la estructura de las moléculas (Chen, 2004).

Ante esta grave crisis del agua a nivel global, hace tiempo que se plantea una gestión ecosistémica del agua, siguiendo los principios del desarrollo sostenible. Ello quiere decir que los recursos hídricos son limitados y que no se pueden mantener sin la conservación de los ecosistemas acuáticos en buen estado, de manera que se haga una gestión eficaz basada en el ahorro, el reuso y la no contaminación del agua, todo ello en una planificación sostenible del territorio y una gestión integrada de las cuencas hidrográficas (Morató et al., 2006).

Hoy día existen tecnologías para el tratamiento de aguas residuales, las cuales dependen en gran medida del tipo de efluente, así como del grado de tratamiento requerido para su reutilización (Nieto, 2011). En la mayoría de los casos se emplean tratamientos convencionales, los cuales incluyen diversas etapas de separación físicas, como cribado, desarenado, sedimentación, separación de grasas y aceites, seguidos de proceso con o sin aireación, para finalmente ser sometidas a etapas de desinfección, como cloración, ozonación o UV. En los últimos años se han desarrollado opciones que proyectan una alta viabilidad en su aplicación, como oxidación química $\left(\mathrm{O}_{3}, \mathrm{O}_{3} / \mathrm{H}_{2} \mathrm{O}_{2}, \mathrm{H}_{2} \mathrm{O}_{2} / \mathrm{Fe}^{+2}\right)$, fotocatálisis $\left(\mathrm{TiO}_{2} / \mathrm{UV}\right.$, foto-Fenton), degradación fotoquímica $\left(\mathrm{O}_{3} / \mathrm{UV}, \mathrm{O}_{3} / \mathrm{H}_{2} \mathrm{O}_{2}\right)$, o bien la combinación de éstos (electro-Fenton; fotoelectro-Fenton, electrocoagulación $/ \mathrm{O}_{3}$ (Rivera, 2016)

Se han desarrollado muchas técnicas para inmovilizar catalizadores de $\mathrm{TiO}_{2}$ sobre un sustrato sólido, por ejemplo, revestimiento por inmersión de suspensión, revestimiento por pulverización, pulverización catódica, métodos relacionados con sol-gel y deposición electroforética. Además, se han probado diferentes tipos de sustratos; por ejemplo, perlas 
de vidrio, tubos de vidrio, fibra de vidrio, cuarzo, acero inoxidable, aluminio, carbón activado y sílice (Hosseini, 2007).

El proceso de fotocatálisis con luz solar es una tecnología simple empleada para mejorar la calidad del agua y disminuir su carga microbiana (Awag, 2002). La inactivación de microorganismos se debe a la acción de la radiación ultravioleta emitida por la energía solar que sumado al proceso Dip-coating (con Dióxido de Titanio) reducen el tiempo de exposición solar para un tratamiento más efectivo, considerando que el Dióxido de titanio es el semiconductor más frecuente usado en fotocatálisis por su bajo costo, fácil accesibilidad, baja toxicidad, resistencia a la fotocorrosión y a su eficiencia catalítica (Castillo, 2017). La destrucción de los contaminantes mediante el empleo de esta técnica tiene por objeto la formación de radicales hidroxilo que posteriormente tienen un efecto oxidante sobre los contaminantes químicos (Garcés et al., 2004).

Las investigaciones en fotocatálisis incrementaron exponencialmente desde 1972 con el descubrimiento de la hidrólisis electroquímica fotoasistida del agua con un monocristal de Dióxido de titanio como electrodo de trabajo y un contraelectrodo de platino, bajo la aplicación de un potencial químico o electroquímico (Nevárez et al., 2012). En este proceso la oxidación tiene lugar directamente en la superficie de la partícula que se utiliza como catalizador o semiconductor (Dióxido de titanio, entre otros), siendo la radiación solar la única fuente de energía (Bandala et al., 2007). La fotocatálisis heterogénea mediada por Dióxido de Titanio y la foto oxidación mediada por compuestos férricos son dos alternativas atractivas para el tratamiento de aguas (Chen, 2004)

La ubicación del Ecuador en el planeta lo categoriza como un país de privilegio científico respecto al empleo de radiación solar para este tipo de estudios pues la incidencia de radiación solar es perpendicular a nuestra superficie durante todo el año. En el Ecuador la recepción de radiación solar varía dentro del territorio nacional únicamente por condiciones climatológicas locales y que varían de acuerdo a la cercanía o lejanía del sol (Díaz, 2012). En este sentido, la gestión del recurso hídrico se convierte en un mecanismo clave para garantizar que el recurso esté disponible en cantidad y calidad para todos los usuarios que dinamizan su existencia (Delgado, 2017)

Algunos estudios han establecido que una fina capa de dióxido de titanio presenta buena respuesta fotocatalítica, por lo que no es necesario aplicar varios ciclos de inmersión. Adicionalmente establecieron que las películas obtenidas por la técnica Dip-Coating no se desactivan después de la primera inmersión y el posterior lavado(Rivera, 2016). La capa final de dióxido de titanio suele medirse mediante el peso de dicha capa, obtenido a través de una diferencia de pesos entre el peso inicial y final del proceso de impregnación (Acevedo, 2015)

\section{Desarrollo metodológico:}

Se trabajó con cuatro unidades experimentales: dos fueron aplicadas por el método SODIS y las otras dos aplicaron SODIS con Dióxido de Titanio en el método DIPCoating. Todas las unidades de estudio fueron sometidas a condiciones iguales de 
temperatura para determinar la efectividad de cada proceso, cada unidad experimental contó con 4 litros de agua residual distribuidos en 1 litro por unidad experimental. Para la recolección de datos se consideraron dentro de análisis de laboratorio a los parámetros microbiológicos de aguas residuales: coliformes totales, coliformes fecales (Castillo,2017).

\section{Proceso de fotocatálisis (SODIS):}

Se verificaron las condiciones climáticas adecuadas para realizar la prueba SODIS, para su aplicabilidad se emplearon dos botellas de plástico PET de 1 litro con el agua residual y se consideraron dos puntos de muestras provenientes del agua residual de la parroquia Ulba del cantón Baños de Agua Santa-Ecuador. Una vez selladas las botellas, estas fueron expuestas al sol por 180 minutos hasta los $42^{\circ} \mathrm{C}$ durante 12 días consecutivos bajo el esquema expuesto a continuación:

Tabla1

Consideraciones para la prueba SODIS

\begin{tabular}{ccclcc}
\hline & IDENTIFICACIÓN & & \multicolumn{2}{c}{ SODIS } \\
\hline TIPO DE & TIEMPO & TEMPERATU & LÍMITE & P1 & P2 \\
COLIFORMES & $(\min )$ & RA $\left({ }^{\circ} \mathbf{C}\right)$ & MÁXIMO & NMP $/ 100$ & NMP $/ 100$ \\
& & & NMP $/ 100 \mathrm{ml}$ & $\mathrm{ml}$ & $\mathrm{ml}$ \\
\hline
\end{tabular}

Fuente: Castillo, (2017)

De la Tabla 1 se denota que P1 y P2 representan la carga microbiana presente en los dos puntos de muestreo del agua residual (NMP/100 ml). Se realizó el conteo de coliformes fecales y totales en Placas Compact Dry EC.

\section{Procedimiento combinado SODIS con Dip - Coating}

\subsection{Inmovilización del Dióxido de Titanio en varillas de vidrio:}

Para la preparación de las varillas de vidrio se pesaron 22 varillas de vidrio de boro silicato de $6 \mathrm{~mm}$ para proceder a su raspado y lavado con la finalidad de incrementar la rugosidad de su superficie, dejándolas secar a $70^{\circ} \mathrm{C}$ durante 2 horas para finalmente ser pesadas.

Para la preparación de la solución de dióxido de titanio se preparó $100 \mathrm{ml}$ de una solución de Dióxido de titanio con agua desmineralizada al 5\% dispuesta en baño ultrasónico por 30 minutos.

La disposición del dióxido de titanio en las varillas de vidrio fue en una capa uniforme de dióxido de titanio, con la ayuda de una bureta de $25 \mathrm{ml}$ sumergidas cada una de ellas en la solución preparada dejándolas secar a $35^{\circ} \mathrm{C}$ por 2 horas y repitiendo este proceso hasta por 12 veces para lograr el espesor de la capa del catalizador adecuado.

El recubrimiento obtenido en las varillas fue calcinado en un horno a $300{ }^{\circ} \mathrm{C}$ durante 4 horas. Al finalizar con la inmovilización del Dióxido de titanio en las varillas de vidrio mediante Dip-Coating se procedió a tomar el peso final de cada una de estas. 


\subsection{Desinfección por radiación solar:}

Las muestras de agua residual para este método fueron dispuestas en dos botellas de plástico PET selladas y agitadas durante 1 minuto.

Cinco varillas de vidrio de boro silicato de $6 \mathrm{~mm}$ inmovilizadas previamente con Dióxido de titanio se introdujeron en cada uno de los envases exponiéndolas en reposo a radiación UV-A (luz solar) por un lapso promedio de 60 minutos. Posterior a este tiempo los envases se agitaron durante 20 segundos para obtener una muestra de cada una de ellas para realizar en ellas sus correspondientes análisis físicos químicos y microbiológicos (Castillo,2017)..

\subsection{Reducción de coliformes de aguas residuales:}

Para la determinación, cuantificación y diferenciación de coliformes se empleó un procedimiento seguro con placas Compact Dry EC compuestas por dos sustratos enzimáticos cromógenos que son: Magenta-GAL y X-Gluc.

Para cada uno de los envases PET utilizados tanto en el método SODIS y SODIS/Dip coating se prepararon 3 disoluciones $\left(10^{-1}, 10^{-2}\right.$ y $\left.10^{-3}\right)$ con agua de peptona en tubos de ensayo que fueron homogenizados y de los cuales se extrajeron $1 \mathrm{ml}$ de muestra para el análisis microbiológico en la cuantificación de coliformes totales y fecales incubadas 24 horas a $35^{\circ} \mathrm{C}$ (Castillo,2017)..

Para el análisis estadístico se realizó la prueba de Kruskal - Wallis para conocer el proceso fotocatalítico con mayor nivel de reducción de coliformes de las aguas residuales.

\section{Análisis de los resultados:}

\section{Proceso de fotocatálisis (SODIS):}

En la presente tabla se encuentran representados los valores adquiridos de coliformes fecales y totales de cada una de las muestras después de ser expuestos a la radiación solar en intervalos de tiempo:

Tabla2

Resultados de la prueba SODIS al 1 día de tratamiento

\begin{tabular}{lccccc}
\hline \multicolumn{2}{c}{ IDENTIFICACIÓN } & \multicolumn{2}{c}{ SODIS } \\
\hline $\begin{array}{c}\text { TIPO DE } \\
\text { COLIFORMES }\end{array}$ & $\begin{array}{c}\text { TIEMPO } \\
\text { (min) }\end{array}$ & $\begin{array}{c}\text { TEMPERA } \\
\text { TURA }\left({ }^{\circ} \mathrm{C}\right)\end{array}$ & $\begin{array}{c}\text { LÍMITE } \\
\text { MÁXIMO } \\
\text { NMP/100ml }\end{array}$ & $\begin{array}{c}\text { P1 } \\
\text { NMP/1 } \\
00 \mathrm{ml}\end{array}$ & $\begin{array}{c}\text { P2 } \\
\text { NMP/1 } \\
00 \mathrm{ml}\end{array}$ \\
\hline Fecales & 0 & & 2000 & 24000 & 24000 \\
Totales & 0 & 18,1 & $\mathrm{~N} / \mathrm{E}$ & 20850 & 19220 \\
Fecales & & & 2000 & 19200 & 19450 \\
Totales & 60 & 26 & $\mathrm{~N} / \mathrm{E}$ & 19800 & 18635 \\
Fecales & 120 & 30 & 2000 & 18150 & 18350
\end{tabular}


Tabla2

Resultados de la prueba SODIS al 1 día de tratamiento (continuación)

\begin{tabular}{cccccc}
\hline & \multicolumn{2}{c}{ IDENTIFICACIÓN } & \multicolumn{2}{c}{ SODIS } \\
\hline $\begin{array}{c}\text { TIPO DE } \\
\text { COLIFORMES }\end{array}$ & $\begin{array}{c}\text { TIEMPO } \\
\text { (min) }\end{array}$ & $\begin{array}{c}\text { TEMPERA } \\
\left.\text { TURA ( }{ }^{\circ} \mathrm{C}\right)\end{array}$ & $\begin{array}{c}\text { LÍMITE } \\
\text { MÁXIMO } \\
\text { NMP/100ml }\end{array}$ & $\begin{array}{c}\text { P1 } \\
\text { NMP/1 } \\
00 \mathrm{ml}\end{array}$ & $\begin{array}{c}\text { P2 } \\
\text { NMP/1 } \\
00 \mathrm{ml}\end{array}$ \\
\hline Totales & 120 & 30 & N/E & 18750 & 15812 \\
Fecales & & & 2000 & 17100 & 17200 \\
Totales & 180 & 42 & N/E & 17700 & 13000 \\
\hline
\end{tabular}

Fuente: (Castillo, 2017).

Al someter las muestras a la radiación solar se obtuvo un resultado favorable a 180 minutos y $42{ }^{\circ} \mathrm{C}$, el cual nos indica que en el punto 1 hubo $17100 \mathrm{NMP} / 100 \mathrm{ml} \mathrm{de}$ coliformes fecales y 17700 de coliformes totales NMP/100 ml; en el punto 2 hubo 17200 NMP/100 ml de coliformes fecales y 13000 de coliformes totales NMP/100 ml.

\section{Figura 1}

Número de coliformes reducidas mediante SODIS

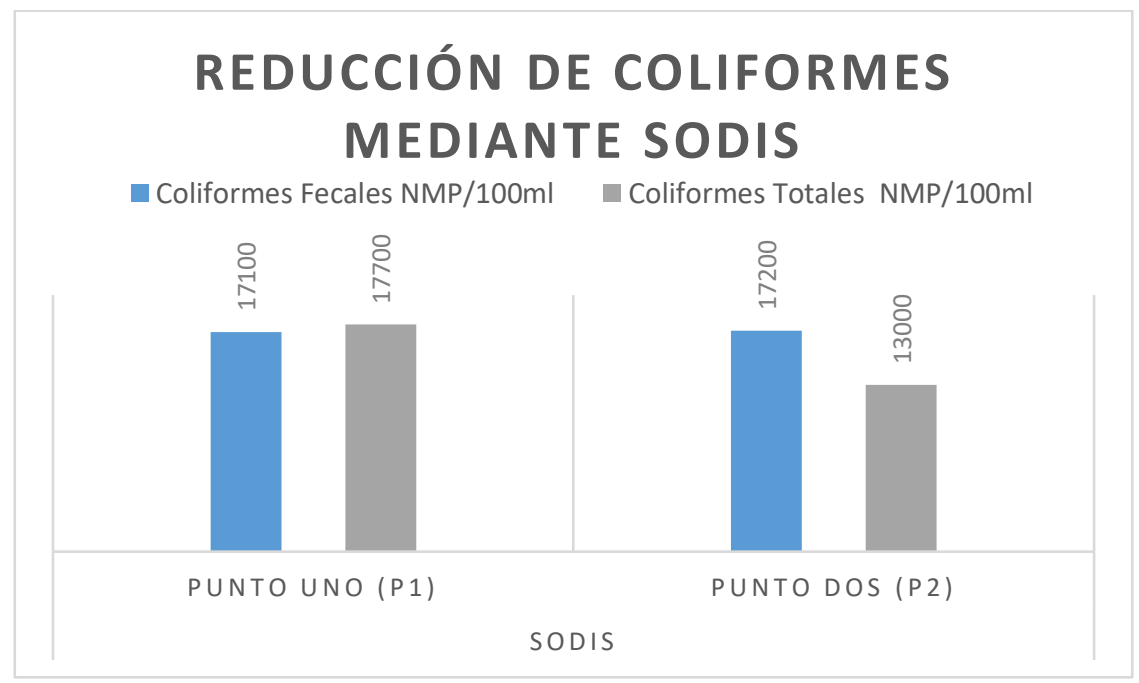

Fuente: Castillo, (2017)

En la tabla 3 se encuentran representados los resultados obtenidos al realizar un análisis para identificar el número de coliformes fecales y totales presentes en las muestras del punto 1 y del punto 2 después de haber transcurrido 12 días de la aplicación del proceso fotocatalítico SODIS.

Tabla3

Resultados para la prueba SODIS a los 12 dias de tratamiento

\begin{tabular}{|c|c|c|c|c|}
\hline \multicolumn{2}{|c|}{ IDENTIFICACIÓN } & \multirow[b]{2}{*}{$\begin{array}{c}\text { LÍMITE } \\
\text { MÁXIMO } \\
\text { NMP/100ml }\end{array}$} & \multicolumn{2}{|c|}{ SODIS } \\
\hline $\begin{array}{c}\text { TIPO DE } \\
\text { COLIFORMES }\end{array}$ & $\begin{array}{l}\text { TIEMPO } \\
\text { (días) }\end{array}$ & & $\begin{array}{c}\text { P1 } \\
\text { NMP/1 } \\
00 \mathrm{ml}\end{array}$ & $\begin{array}{c}\mathbf{P 2} \\
\text { NMP/1 } \\
00 \mathrm{ml}\end{array}$ \\
\hline Fecales & 12 & 2000 & 7000 & 2000 \\
\hline Totales & 12 & N/E & 14000 & 11700 \\
\hline
\end{tabular}

Fuente: (Castillo, 2017). 
Al haber transcurrido un período de 12 días se tuvo una menor cantidad de coliformes fecales y coliformes totales, lo que indica que al transcurrir el tiempo el proceso SODIS es más efectivo.

Figura 2

Número de coliformes reducidas mediante SODIS a los 12 días de tratamiento

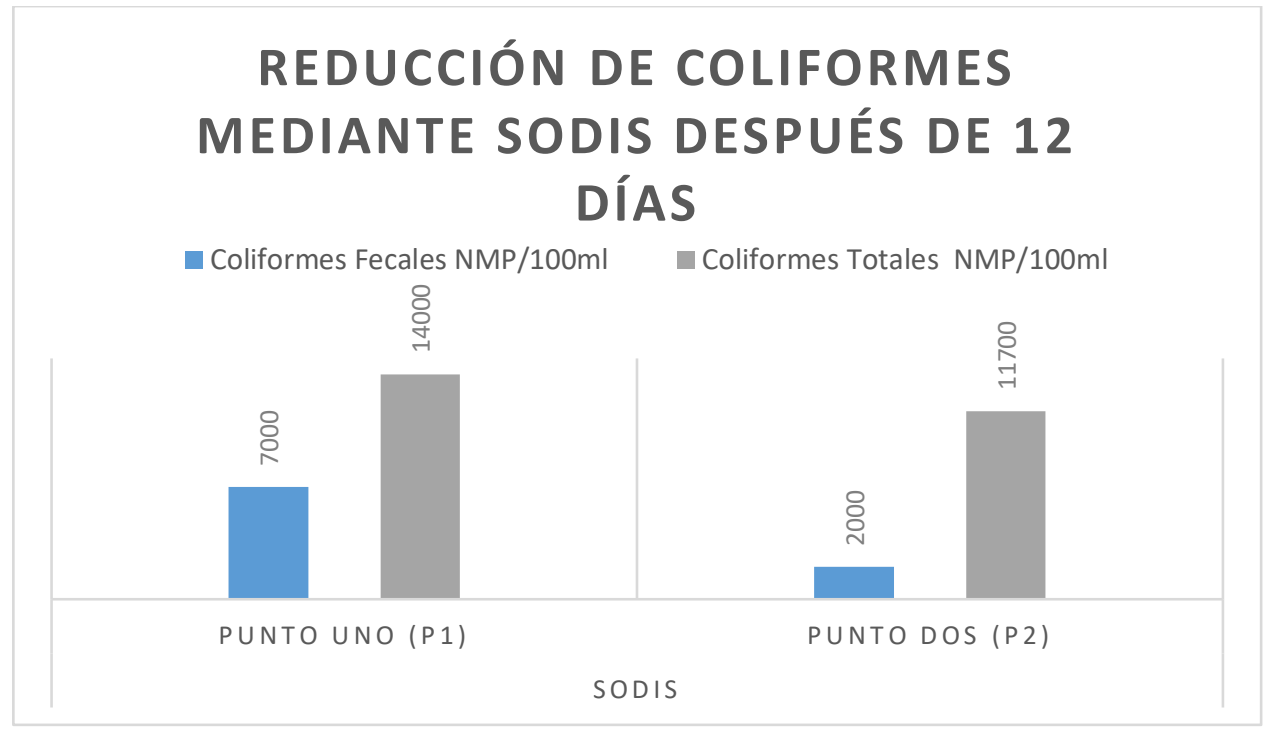

Fuente: Castillo, (2017)

A continuación, se muestra la identificación del número de coliformes en las placas Compact Dry EC para SODIS

Figura 3

Identificación de coliformes SODIS

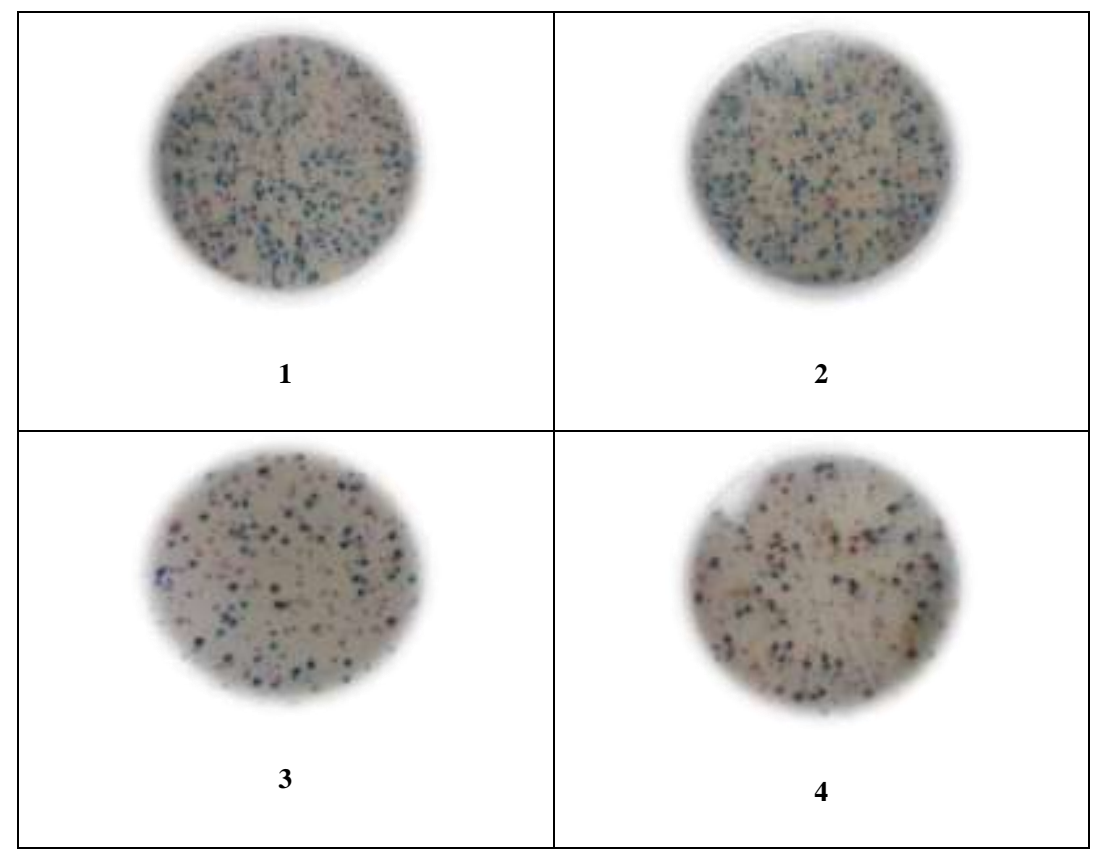

Fuente: Castillo, (2017) 
En la ilustración 1 se pueden diferenciar a las coliformes fecales que poseen coloración azul y a coliformes totales color rojo; en la imagen 1 perteneciente al punto 1 de muestreo se pudieron observar coliformes fecales y totales aproximadamente en las mismas proporciones, en el punto 2 de muestreo (imagen 2) se pudo observar mayor cantidad coliformes fecales que de coliformes totales (Castillo,2017).

Al transcurrir 12 días, en el punto 1 (imagen 3) y en el punto 2 (imagen 4), se encontraron una reducción significativa de coliformes fecales y en menor cantidad coliformes totales; esto producto de las condiciones climáticas y la ubicación en la que se encontraban las muestras (Castillo,2017).

\section{Proceso de fotocatálisis (SODIS) con DIP- coating:}

En la tabla 4 se encuentran representados los valores adquiridos en cada repetición aplicada para inmovilizar Dióxido de titanio (gramos /varilla) en 22 varillas de Boro silicato mediante el método Dip-Coating con doce repeticiones después de dos horas de actuación

Tabla 4

Inmovilización del dióxido de titanio en las varillas de vidrio

\begin{tabular}{|c|c|c|c|c|c|c|c|c|c|c|c|c|c|}
\hline $\begin{array}{l}\mathrm{N}^{\circ} \text { de } \\
\text { Varilla }\end{array}$ & $\mathbf{P i}(\mathrm{g})$ & $\begin{array}{l}\text { R1 } \\
\text { (g) }\end{array}$ & $\begin{array}{l}\text { R2 } \\
\text { (g) }\end{array}$ & $\begin{array}{l}\text { R3 } \\
\text { (g) }\end{array}$ & $\begin{array}{l}\text { R4 } \\
\text { (g) }\end{array}$ & $\begin{array}{l}\text { R5 } \\
\text { (g) }\end{array}$ & $\begin{array}{l}\text { R6 } \\
\text { (g) }\end{array}$ & $\begin{array}{l}\text { R7 } \\
\text { (g) }\end{array}$ & $\begin{array}{l}\text { R8 } \\
\text { (g) }\end{array}$ & $\begin{array}{l}\text { R9 } \\
\text { (g) }\end{array}$ & $\begin{array}{l}\text { R10 } \\
\text { (g) }\end{array}$ & $\begin{array}{l}\text { R11 } \\
\text { (g) }\end{array}$ & $\begin{array}{c}\text { R12 } \\
\text { (g) }\end{array}$ \\
\hline 1 & 12,52 & 12,50 & 12,48 & 12,50 & 12,52 & 12,50 & 12,52 & 12,52 & 12,52 & 12,52 & 12,56 & 12,52 & 12,54 \\
\hline 2 & 12,54 & 12,54 & 12,56 & 12,58 & 12,60 & 12,62 & 12,66 & 12,64 & 12,64 & 12,64 & 12,64 & 12,62 & 12,62 \\
\hline 3 & 12,62 & 12,60 & 12,60 & 12,60 & 12,60 & 12,60 & 12,62 & 12,60 & 12,60 & 12,62 & 12,64 & 12,58 & 12,64 \\
\hline 4 & 12,64 & 12,62 & 12,62 & 12,64 & 12,62 & 12,60 & 12,64 & 12,62 & 12,62 & 12,64 & 12,64 & 12,62 & 12,66 \\
\hline 5 & 12,64 & 12,64 & 12,66 & 12,68 & 12,64 & 12,64 & 12,66 & 12,64 & 12,64 & 12,66 & 12,66 & 12,62 & 12,66 \\
\hline 6 & 12,70 & 12,70 & 12,70 & 12,70 & 12,70 & 12,70 & 12,72 & 12,70 & 12,68 & 12,72 & 12,72 & 12,66 & 12,72 \\
\hline 7 & 12,70 & 12,70 & 12,70 & 12,70 & 12,72 & 12,70 & 12,72 & 12,70 & 12,72 & 12,74 & 12,70 & 12,70 & 12,72 \\
\hline 8 & 12,72 & 12,72 & 12,70 & 12,72 & 12,72 & 12,72 & 12,74 & 12,72 & 12,74 & 12,74 & 12,72 & 12,72 & 12,74 \\
\hline 9 & 12,72 & 12,74 & 12,74 & 12,76 & 12,74 & 12,74 & 12,74 & 12,74 & 12,74 & 12,76 & 12,74 & 12,72 & 12,74 \\
\hline 10 & 12,78 & 12,80 & 12,78 & 12,78 & 12,78 & 12,78 & 12,78 & 12,78 & 12,76 & 12,80 & 12,78 & 12,76 & 12,80 \\
\hline 11 & 12,80 & 12,80 & 12,80 & 12,78 & 12,80 & 12,80 & 12,80 & 12,80 & 12,78 & 12,82 & 12,82 & 12,78 & 12,82 \\
\hline 12 & 12,80 & 12,80 & 12,80 & 12,80 & 12,80 & 12,80 & 12,82 & 12,80 & 12,80 & 12,82 & 12,84 & 12,78 & 12,82 \\
\hline 13 & 12,82 & 12,84 & 12,84 & 12,84 & 12,84 & 12,82 & 12,84 & 12,84 & 12,82 & 12,86 & 12,84 & 12,80 & 12,84 \\
\hline 14 & 12,84 & 12,84 & 12,84 & 12,84 & 12,84 & 12,82 & 12,86 & 12,84 & 12,82 & 12,86 & 12,84 & 12,82 & 12,86 \\
\hline 15 & 12,84 & 12,84 & 12,84 & 12,84 & 12,84 & 12,84 & 12,86 & 12,84 & 12,86 & 12,86 & 12,86 & 12,84 & 12,86 \\
\hline 16 & 12,84 & 12,84 & 12,84 & 12,84 & 12,84 & 12,86 & 12,86 & 12,84 & 12,86 & 12,86 & 12,86 & 12,86 & 12,88 \\
\hline 17 & 12,88 & 12,88 & 12,90 & 12,92 & 12,94 & 12,96 & 12,98 & 12,98 & 12,98 & 13,00 & 12,98 & 13,00 & 12,90 \\
\hline 18 & 12,90 & 12,90 & 12,92 & 12,90 & 12,90 & 12,90 & 12,92 & 12,90 & 12,90 & 12,92 & 12,90 & 12,86 & 12,92 \\
\hline 19 & 13,00 & 13,00 & 13,02 & 13,02 & 13,02 & 13,00 & 13,02 & 13,02 & 13,00 & 12,98 & 13,04 & 13,02 & 13,02 \\
\hline 20 & 13,02 & 13,02 & 13,04 & 13,04 & 13,04 & 13,00 & 13,04 & 13,02 & 13,00 & 13,04 & 13,04 & 13,02 & 13,04 \\
\hline 21 & 13,08 & 13,06 & 13,08 & 13,08 & 13,08 & 13,06 & 13,08 & 13,08 & 13,08 & 13,10 & 13,08 & 13,04 & 13,10 \\
\hline 22 & 13,08 & 13,10 & 13,10 & 13,08 & 13,10 & 13,10 & 13,10 & 13,10 & 13,12 & 13,10 & 13,10 & 13,10 & 13,12 \\
\hline
\end{tabular}

Fuente: Castillo, (2017)

La variación de los pesos en las varillas fue consecuencia de aspectos como su origen, varillas sometidas a un proceso de secado en horno $\left(35^{\circ} \mathrm{C}\right)$ y del lavado con agua destilada, ocasionando que partículas de Dióxido de titanio se vayan adhiriendo en proporciones distintas en cada una de ellas. Sin embargo, estos resultados no variaron significativamente (Castillo,2017). 
Tabla 5

Peso final de las varillas de vidrio con dióxido de titanio inmovilizado en ellas

\begin{tabular}{|c|c|c|c|c|c|}
\hline $\begin{array}{l}N^{\circ} \text { de } \\
\text { Varilla }\end{array}$ & $\begin{array}{l}\text { Peso de la } \\
\text { varilla con } \\
\text { rugosidad (g) }\end{array}$ & $\begin{array}{l}\text { Longitud de } \\
\text { la varilla }(\mathrm{cm})\end{array}$ & $\begin{array}{l}\text { Diámetro de } \\
\text { la varilla } \\
\text { (mm) }\end{array}$ & $\begin{array}{l}\text { Peso de } \\
\mathrm{TiO}_{2} \text { en } \\
\quad \mathrm{g}\end{array}$ & $\begin{array}{l}\text { Peso final } \\
\text { de la varilla } \\
\text { (g) }\end{array}$ \\
\hline 1 & 12,52 & 20 & 6 & 0,02 & 12,54 \\
\hline 2 & 12,54 & 20 & 6 & 0,08 & 12,62 \\
\hline 3 & 12,62 & 20 & 6 & 0,02 & 12,64 \\
\hline 4 & 12,64 & 20 & 6 & 0,02 & 12,66 \\
\hline 5 & 12,64 & 20 & 6 & 0,02 & 12,66 \\
\hline 6 & 12,70 & 20 & 6 & 0,02 & 12,72 \\
\hline 7 & 12,70 & 20 & 6 & 0,02 & 12,72 \\
\hline 8 & 12,72 & 20 & 6 & 0,02 & 12,74 \\
\hline 9 & 12,72 & 20 & 6 & 0,02 & 12,74 \\
\hline 10 & 12,78 & 20 & 6 & 0,02 & 12,80 \\
\hline 11 & 12,80 & 20 & 6 & 0,02 & 12,82 \\
\hline 12 & 12,80 & 20 & 6 & 0,02 & 12,82 \\
\hline 13 & 12,82 & 20 & 6 & 0,02 & 12,84 \\
\hline 14 & 12,84 & 20 & 6 & 0,02 & 12,86 \\
\hline 15 & 12,84 & 20 & 6 & 0,02 & 12,86 \\
\hline 16 & 12,84 & 20 & 6 & 0,04 & 12,88 \\
\hline 17 & 12,88 & 20 & 6 & 0,02 & 12,90 \\
\hline 18 & 12,90 & 20 & 6 & 0,02 & 12,92 \\
\hline 19 & 13,00 & 20 & 6 & 0,02 & 13,02 \\
\hline 20 & 13,02 & 20 & 6 & 0,02 & 13,04 \\
\hline 21 & 13,08 & 20 & 6 & 0,02 & 13,10 \\
\hline 22 & 13,08 & 20 & 6 & 0,04 & 13,12 \\
\hline
\end{tabular}

Fuente: Castillo, (2017)

La relación del peso adherido se basa en una adhesión de 0,04 gramos en la varilla número 22 y número 16 con un resultado sobresaliente en la varilla número 2 en la que la adhesión fue de 0.08 gramos (Castillo,2017)

De las 22 varillas sometidas al tratamiento se tomaron 10 varillas al azar cuyo peso de adhesión fue de 0,02 gramos. Las varillas inmovilizadas con dióxido de titanio generalmente mantuvieron el mismo peso, no existió pérdida del reactivo (Castillo,2017). 


\section{Tabla 6}

Varillas de vidrio con dióxido de titanio seleccionadas al azar, de acuerdo al peso final adquirido para someterlas al proceso SODIS/Dip-Coating

\begin{tabular}{|c|c|c|c|c|c|}
\hline $\mathbf{N}^{\circ}$ de Varilla & $\begin{array}{l}\text { Peso final de } \\
\text { la varilla (g). }\end{array}$ & $\begin{array}{l}\text { Longitud de } \\
\text { la varilla } \\
\text { (cm). }\end{array}$ & $\begin{array}{l}\text { Diámetro de } \\
\text { la varilla } \\
\text { (mm). }\end{array}$ & $\begin{array}{l}\text { Peso de } \mathrm{TiO}_{2} \\
\text { (g). }\end{array}$ & $\begin{array}{c}\text { Peso varilla } \\
\mathrm{TiO}_{2} \text { después } \\
\text { de } 12 \text { días }\end{array}$ \\
\hline \multicolumn{6}{|c|}{ Punto de muestreo 1 (P1). } \\
\hline 1 & 12,7481 & 20 & 6 & 0,02 & 12,7482 \\
\hline 2 & 12,7512 & 20 & 6 & 0,02 & 12,7612 \\
\hline 3 & 12,7623 & 20 & 6 & 0,02 & 12,7801 \\
\hline 4 & 12,7753 & 20 & 6 & 0,02 & 12,8002 \\
\hline 5 & 12,8212 & 20 & 6 & 0,02 & 12,8213 \\
\hline \multicolumn{6}{|c|}{ Punto de muestreo 2 (P2). } \\
\hline 6 & 12,8404 & 20 & 6 & 0,02 & 12,8404 \\
\hline 7 & 12,8790 & 20 & 6 & 0,02 & 12,8801 \\
\hline 8 & 12,9396 & 20 & 6 & 0,02 & 12,9400 \\
\hline 9 & 13,1143 & 20 & 6 & 0,02 & 13,1201 \\
\hline 10 & 13,1375 & 20 & 6 & 0,02 & 13,1400 \\
\hline
\end{tabular}

Fuente: Castillo, (2017)

En la tabla 7 se encuentran representados los valores adquiridos de coliformes fecales y coliformes totales presentes en cada una de las muestras después de ser expuestos a la radiación solar con intervalos de tiempo correspondiente al proceso fotocatalítico SODIS/Dip-Coating (Castillo,2017)

Tabla 7

Resultados del análisis microbiológico de SODIS/DIP- Coating

\begin{tabular}{|c|c|c|c|c|c|}
\hline \multicolumn{3}{|c|}{ IDENTIFICACIÓN } & \multirow[b]{2}{*}{$\begin{array}{c}\text { LÍMITE } \\
\text { MÁXIMO } \\
\text { (NMP/100 ml) }\end{array}$} & \multicolumn{2}{|c|}{ SODIS/Dip-Coating } \\
\hline $\begin{array}{c}\text { TIPO DE } \\
\text { COLIFORMES }\end{array}$ & $\begin{array}{l}\text { TIEMPO } \\
(\min )\end{array}$ & $\begin{array}{l}\text { TEMPERATURA } \\
\left({ }^{\circ} \mathrm{C}\right)\end{array}$ & & $\begin{array}{c}\text { P1 } \\
\text { (NMP/100 } \\
\text { ml) }\end{array}$ & $\begin{array}{c}\text { P2 } \\
\text { (NMP/100 } \\
\mathrm{ml} \text { ) }\end{array}$ \\
\hline Fecales & & & 2000 & 24000 & 24000 \\
\hline Totales & 0 & 18,1 & N/E & 20850 & 19220 \\
\hline Fecales & & & 2000 & 13950 & 13475 \\
\hline Totales & 60 & 26 & $\mathrm{~N} / \mathrm{E}$ & 17235 & 15470 \\
\hline Fecales & & & 2000 & 6150 & 9225 \\
\hline Totales & 120 & 30 & $\mathrm{~N} / \mathrm{E}$ & 14620 & 13110 \\
\hline Fecales & & & 2000 & 3900 & 100 \\
\hline Totales & 180 & 42 & $N / E$ & 12500 & 1100 \\
\hline
\end{tabular}

Fuente: Castillo, (2017)

Al someter las muestras a la radiación solar se obtuvo un resultado favorable a 180 minutos y $42{ }^{\circ} \mathrm{C}$, que indicó que en el punto 1 hubieron $3900 \mathrm{NMP} / 100 \mathrm{ml}$ de coliformes fecales y 12500 de coliformes totales NMP/100 ml; mientras que en el punto 2 hubieron $100 \mathrm{NMP} / 100 \mathrm{ml}$ de coliformes fecales y 1100 de coliformes totales NMP/100 ml; donde 
esta última presentó un menor crecimiento microbiano en los rangos de temperatura y tiempo establecidos cómo se indica en la siguiente gráfica (Castillo,2017)

\section{Figura 4}

Número de coliformes reducidas mediante SODIS/ Dip - Coating

\section{REDUCCIÓN DE COLIFORMES \\ MEDIANTE SODIS/DIP-COATING}

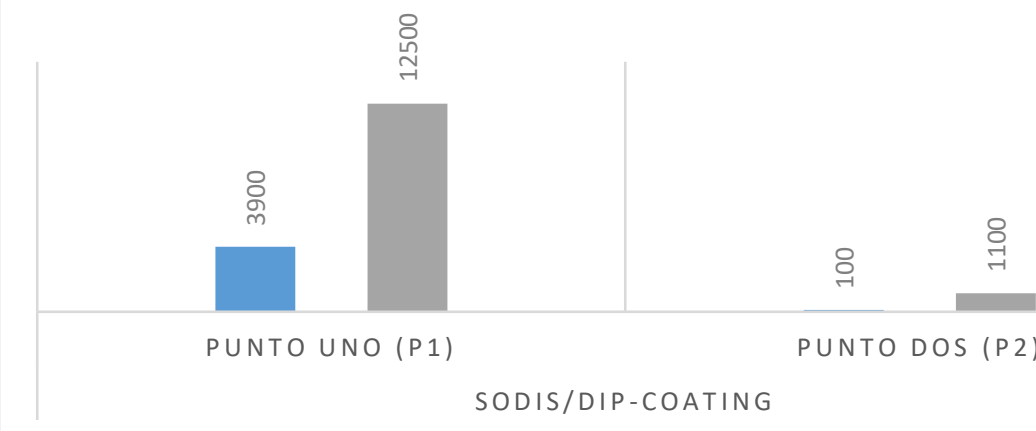

Fuente: Castillo, (2017)

Se evidenció una reducción significativa para los dos puntos de muestreo, pero en el punto dos (P2) existió una reducción mayor de coliformes fecales y coliformes totales en comparación con el punto uno (P1), esto porque las muestras fueron obtenidas de dos puntos distintos y por el grado de incidencia de la luz solar dentro de la cual a mayor grado de incidencia de la luz solar mayor reducción de la carga microbiana. Esta reducción significativa se relaciona directamente con la comprensión de la relación entre múltiples presiones, condiciones y servicios de los ecosistemas acuáticos que ayuda a diseñar medidas para lograr el objetivo del buen estado de los sistemas hídricos considerando los beneficios de invertir en la conservación y restauración de la naturaleza (Grizzetti, 2016)

\section{Figura 5}

Número de coliformes mediante SODIS/ DIP - Coating después de 12 días

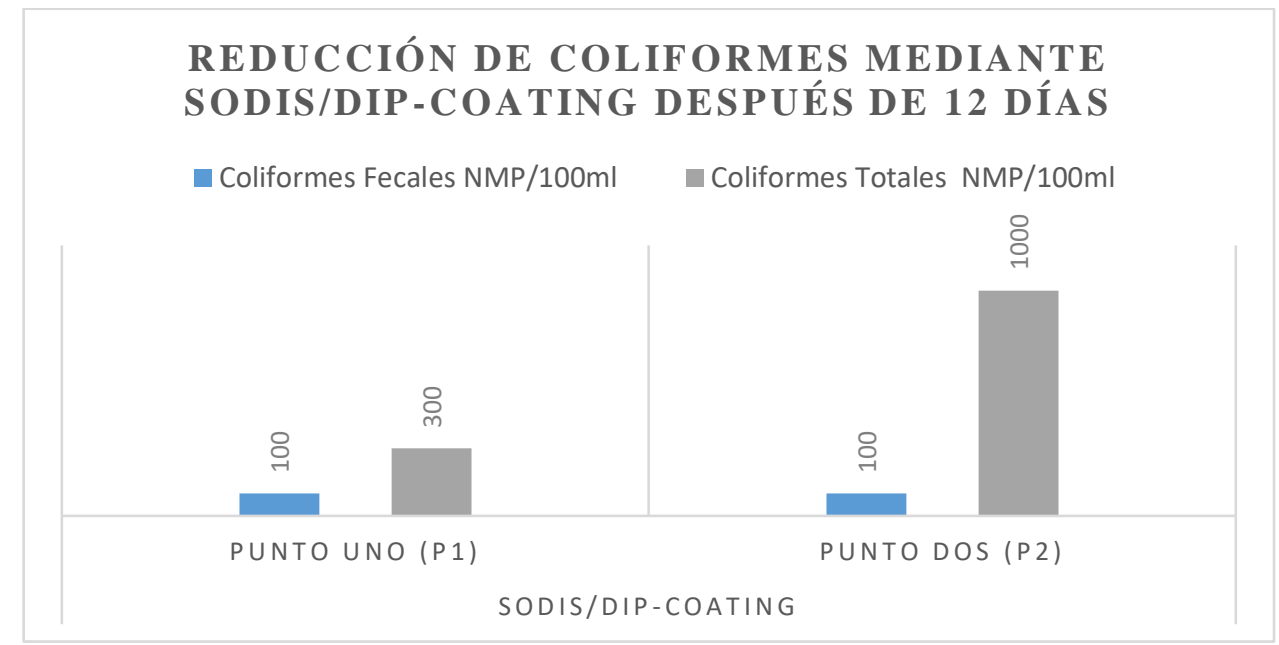

Fuente: Castillo, (2017) 
Existió una reducción significativa en el punto uno (P1) después de 12 días de aplicado el proceso, en cambio en el punto dos (P2) los valores se mantuvieron constantes.

\section{Figura 6}

Identificación de coliformes SODIS/Dip-Coating.

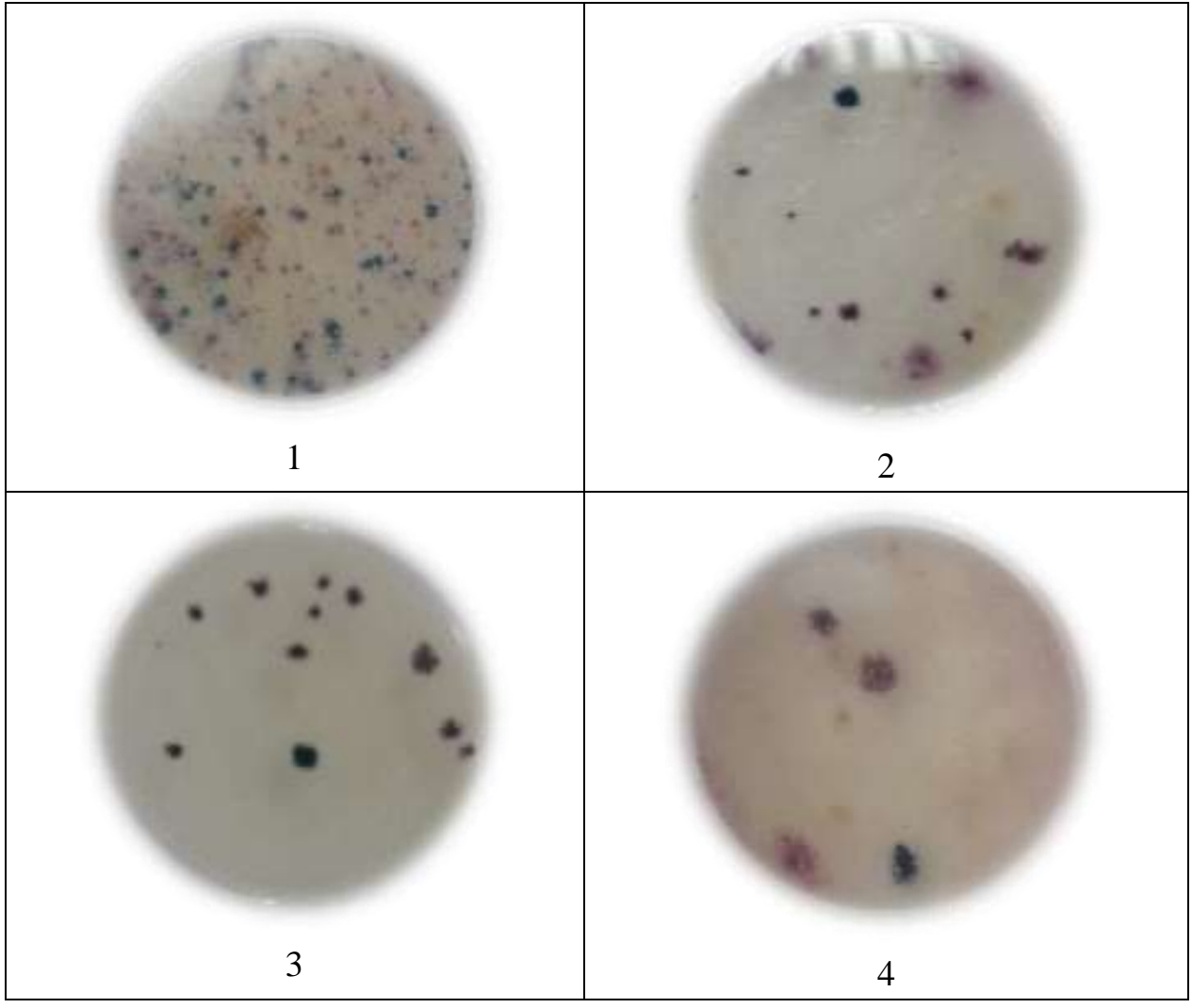

Fuente: Castillo, (2017)

En la ilustración 2 se puede diferenciar a los coliformes fecales con color azul y coliformes totales identificados por el color rojo; en la imagen 1 se puede observar una mayor cantidad de coliformes totales, en el punto 2 (imagen 2) se pudo observar la reducción de coliformes fecales con mayoritaria presencia de coliformes totales. Al transcurrir 12 días, en el punto 1 (imagen 3) se encontró una reducción significativa de coliformes fecales, en el punto 2 (imagen 4 ) existió una reducción significativa tanto en coliformes fecales como en coliformes totales, pero destacando que la reducción principal fue en coliformes fecales (Castillo,2017).

Prueba de Kruskal-Wallis para SODIS/Dip-Coating.

Tabla 8

Rangos para Kruskal-Wallis SODIS/Dip-Coating

\begin{tabular}{llr}
\hline \multicolumn{2}{c}{ Rangos } \\
\hline & TIEMPO_EXPOSICIÓN & N \\
\hline COLIFORMES_SODIS_Ti & 0 & 4 \\
O2 & 60 & 4 \\
\hline
\end{tabular}


Tabla 8

Rangos para Kruskal-Wallis SODIS/Dip-Coating (continuación)

\begin{tabular}{llr}
\hline & \multicolumn{2}{c}{ Rangos } \\
\hline & TIEMPO_EXPOSICIÓN & N \\
\hline \multirow{2}{*}{ COLIFORMES_SODIS_TiO } & 120 & 4 \\
2 & 180 & 4 \\
& Total & 16
\end{tabular}

Fuente: Castillo, (2017)

En la primera columna se ubican la información de coliformes con SODIS/Dip-Coating así como el tiempo de exposición, en la segunda columna el número de casos válidos.

Tabla 9

Prueba de Kruskal-Wallis SODIS/Dip-Coating

Estadísticos de contraste ${ }^{a, b}$

\begin{tabular}{lr}
\hline & COLIFORMES_SODIS_TiO2 \\
\hline Chi-cuadrado & 12,813 \\
gl & 3 \\
Sig. asintót. &, 005
\end{tabular}
a. Prueba de Kruskal-Wallis
b. Variable de agrupación: TIEMPO_EXPOSICIÓN

Como $\mathrm{P}=0,005$ esto representó que existen diferencias en la concentración de coliformes por efecto del tiempo de exposición

\section{Figura 7}

Número de coliformes mediante SODIS/DIP-Coating en relación al tiempo de exposición.

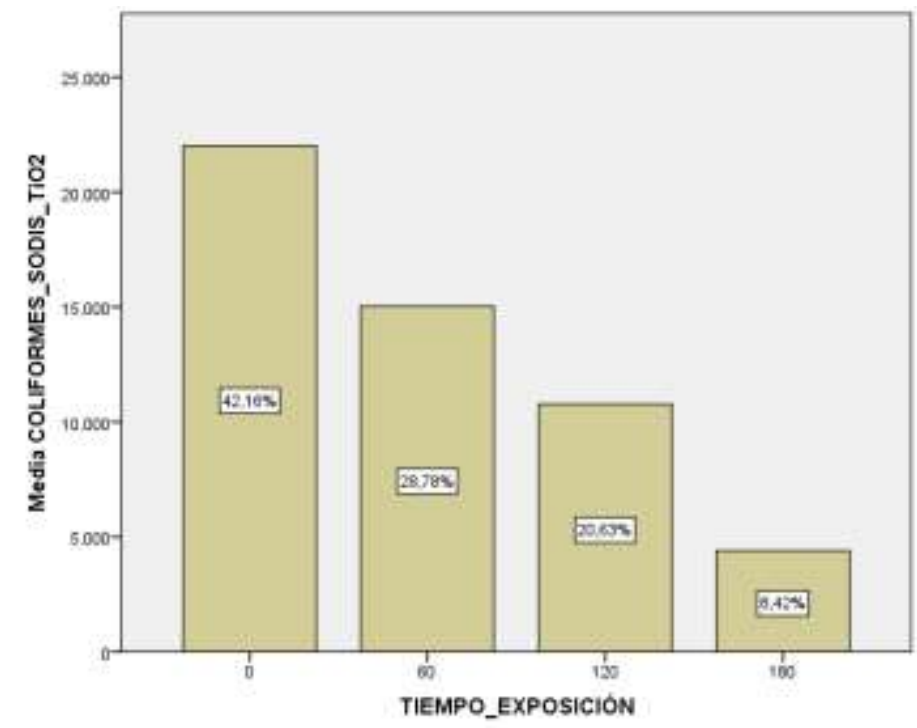

Fuente: Castillo, (2017) 
El gráfico 5 muestra en el eje de las abscisas el tiempo de exposición expresado en minutos ( $\min$ ) y en el eje de las ordenadas el número de coliformes reducidas expresadas en $(\mathrm{NMP} / 100 \mathrm{ml})$ en el cual se empleó el método SODIS/Dip-Coating para reducir coliformes de las aguas residuales. Las barras representan el porcentaje de reducción existente entre el número de coliformes y el tiempo de exposición como resultado de la aplicación del método SODIS/Dip-Coating, obteniendo un resultado favorable a 180 minutos (Castillo,2017).

\section{Conclusiones:}

- De las cuatro unidades experimentales propuestas en el proceso metodológico y con base en el número de coliformes presentes y reducidas en las aguas residuales durante la aplicación de SODIS y SODIS/Dip-Coating las condiciones óptimas para el desarrollo del proceso fueron en el rango de $18.1{ }^{\circ} \mathrm{C} \mathrm{a} 42{ }^{\circ} \mathrm{C}$, tiempo de exposición 180 minutos, incidencia de los rayos ultravioletas de la región A (UVA) que es la radiación ultravioleta natural que llega a la superficie terrestre con una longitud de onda comprendida entre 400 y $320 \mathrm{~nm}$. Dichas condiciones determinaron que el proceso fotocatalítico con luz solar más óptimo sea SODIS/Dip-Coating; donde las coliformes se redujeron más rápidamente y en mayor proporción.

- Se evidenció una reducción significativa, sobre todo en la dilución 10-3, llegando a contabilizar para el caso de coliformes fecales en el P1: 3900 NMP/100ml y P2: 100 NMP/100ml; y para coliformes totales: P1: 12500 NMP/100ml y P2: 1100 $\mathrm{NMP} / 100 \mathrm{ml}$, a condiciones óptimas de tratamiento de $180 \mathrm{~min}$ a $42^{\circ} \mathrm{C}$. Dichos resultados demostraron que bajo las condiciones expuestas y condiciones climáticas adecuadas se da el efecto de reducción máxima de coliformes fecales y totales. En cuanto al proceso de fotocatálisis con luz solar SODIS se pudo observar una reducción menor en comparación al otro proceso aplicado, llegando a contabilizar en el P1: 17100 NMP/100ml y P2: 17200 NMP/100ml de coliformes fecales; P1: $17700 \mathrm{NMP} / 100 \mathrm{ml}$ y P2: $13000 \mathrm{NMP} / 100 \mathrm{ml}$ de coliformes totales, bajo las mismas condiciones expuestas. El proceso de fotocatálisis con luz solar más adecuado para la reducción de coliformes fecales y totales de aguas residuales es SODIS/Dip-Coating.

- La eficiencia del proceso SODIS/ Dip-Coating para reducir coliformes fecales y coliformes totales de las aguas residuales de estudio fue comprobada, pero sobre todo se logró conseguir con ello el aprovechamiento de tecnologías limpias para cumplir con el objetivo propuesto promoviendo el tratamiento terciario de las aguas residuales y así promover el aprovechamiento de la energía solar y la aplicabilidad conjunta de tecnologías coadyuvantes para su desarrollo, en este caso de estudio se empleó el Dióxido de Titanio que tuvo el rol de catalizador del proceso. La fotocatálisis como una tecnología simple, económica, eficaz e innovadora, para el tratamiento de aguas, el atractivo de esta tecnología radica en 
su rentabilidad, facilidad de implementación y uso, y la inversión requerida de capital es mínima, en comparación con otras tecnologías (Gil, 2002).

\section{Bibliografía:}

Garcés Giraldo, L. F., Mejía Franco , E. A., y Santamaría Arango, J. J. (2004). La fotocatálisis como alternativa para el tratamiento. Revista Lasallista de Investigación, 83-92.

Acevedo Dávila, A. (2015). EStudio de la fotocatálisis heterogénea solar para tratar un efluente contaminado con productos farmacéuticos: Ibuprofeno, Diclofenaco, Sulfametoxazol Obtenido de http://bibdigital.epn.edu.ec/bitstream/15000/10366/3/CD-6165.pdf

Awag, S. (2002). SODIS (Sauberes trinkwasser für alle). Obtenido de SODIS (Sauberes trinkwasser für alle): http://www.sodis.ch/methode/anwendung/ausbildungsmaterial/dokumente_mate rial/manual_s.pdf

Castillo, E. (2017). Procesos de fotocatálisis con luz solar y el método DIP - coating para la reducción de coliformes de aguas residuales del barrio la ciénega con desembocadura en el embalse Agoyán

Chen, C. (2004). Photocatalysis by Titanium Dioxide and Polyoxometalate/TiO2 Cocatalysts.Intermediates and Mechanistic Study. Enviromental Science \& Technology, 329-337.

Delgado García, S. M., Trujillo González, J. M., y Torres Mora, M. A. (2017). Gestión del agua en comunidades rurales; caso de estudio Cuenca del río Guayuriba, MetaColombia. Luna Azul, 59-70.

Díaz - Cuenca, E., Alvarado-Granados, A, y Camacho- Calzada, K. E. (2012). El tratamiento de agua residual doméstica para el desarrollo local sostenible: el caso de la técnica delsistema unitario de tratamiento de aguas, nutrientes y energía (SUTRANE) en San Miguel Almaya,México. Quivera, 78-97.

Gil, Edison (2002). Fotocatálisis: una alternativa viable para la eliminación de compuestos orgánicos. Revista Universidad de EAFIT. Medellín, 59-64.

Grizzetti , B., Lanzanova , D., Liquete C, C., Reynaud, A., y Cardoso, A. (2016). Assessing water ecosystem services for water resource management. Environ Sci Policy, 194-203.

Hosseini S.N. (2007). Inmobilization of $\mathrm{TiO} 2$ on Perlite Granuels for Photocatalitic ELSEVIER. Degradation of Phenol. Science Direct. Applied Catalysis B: Environmental 74, 53-62 
Morató, J., Gris, A., Carneiro, A., y Pastor, R. (2006). Tecnologías sostenibles para la potabilización y el tratamiento de aguas residuales. Revista Lasallista de Investigación, 19-29.

Nevárez, M., Espinoza, P., Quiroz, F., y Ohtani, B. (2012). Fotocatálisis: inicio, actualidad y perspectivas a través del TiO2. Avances en Química, 45-49.

Nieto, N. (2011). La gestión del agua: tensiones globales y latinoamericanas. . Política y cultura, 157-176.

Restrepo, G. M., Ríos, L. A., \& Marín , J. M. (2008). EVALUACIÓN DEL TRATAMIENTO FOTOCATALÍTICO DE AGUAS RESIDUALES INDUSTRIALES EMPLEANDO ENERGÍA SOLAR . Dyna, 145-153.

Rivera Gallardo, P. (2016). Estudio preliminar del uso de la fotoxidación y fotocatálisis como alternativa para el tratamiento de las aguas de lavado de aceite y aceituna, utilizando $\mathrm{TiO} 2$ como catalizador, $\mathrm{H} 2 \mathrm{O} 2$ como oxidante y luz UV (Tesis de pregrado, Universidad de Concepción Chile). eula. http://www.eula.cl/giba/wpcontent/uploads/2017/09/tesis-pilar-rivera-2016.pdf 


\section{PARA CITAR EL ARTÍCULO INDEXADO.}

Godoy Ponce, S. C., González García, J. C., León Chimbolema, J. G., \& Castillo Hidalgo, E. D. (2021). Evaluación de la capacidad de reacción de coliformes de aguas residuales a la fotocatálisis con técnicas de recubrimiento por inmersión . AlfaPublicaciones, 3(3.1), 357-374. https://doi.org/10.33262/ap.v3i3.1.102

\section{Ciencia \\ Ligital \\ Editorial}

El artículo que se publica es de exclusiva responsabilidad de los autores y no necesariamente reflejan el pensamiento de la Revista Alfa Publicaciones.

El artículo queda en propiedad de la revista y, por tanto, su publicación parcial y/o total en otro medio tiene que ser autorizado por el director de la Revista Alfa Publicaciones.
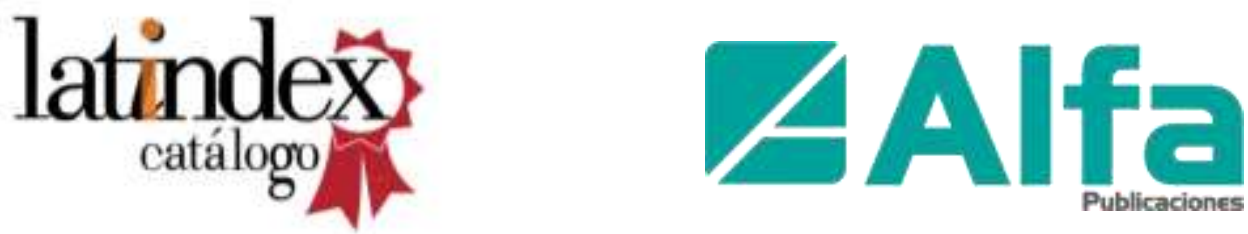